\title{
Chemical scissors cut phosphorene nanostructures
}

\author{
Xihong Peng ${ }^{1}$ and Qun Wei ${ }^{1,2}$ \\ ${ }^{1}$ School of Letters and Sciences, Arizona State University, Mesa, Arizona 85212, USA \\ ${ }^{2}$ School of Physics and Optoelectronic Engineering, Xidian University, Xi'an, 710071, People's \\ Republic of China \\ E-mail: xihong.peng@asu.edu
}

Received 21 August 2014, revised 15 October 2014

Accepted for publication 23 October 2014

Published 28 November 2014

Materials Research Express 1 (2014) 045041

doi:10.1088/2053-1591/1/4/045041

\begin{abstract}
Phosphorene, a recently fabricated two-dimensional puckered honeycomb structure of phosphorus, showed promising properties for applications in nanoelectronics. In this work, we report a chemical scissors effect on phosphorene, using first-principles method. It was found that chemical species, such as $\mathrm{H}, \mathrm{OH}$, $\mathrm{F}$, and $\mathrm{Cl}$, can act as scissors to cut phosphorene. Phosphorus nanochains and nanoribbons can be obtained. The scissors effect results from the strong bonding between the chemical species and phosphorus atoms. Other species such as $\mathrm{O}, \mathrm{S}$ and Se fail to cut phosphorene nanostructures due to their weak bonding with phosphorus. The electronic structures of the produced P-chains reveal that the hydrogenated chain is an insulator while the pristine chain is a one-dimensional Dirac material, in which the charge carriers are massless fermions travelling at an effective speed of light $\sim 8 \times 10^{5} \mathrm{~m} \mathrm{~s}^{-1}$. The obtained zigzag phosphorene nanoribbons show either metallic or semiconducting behaviors, depending on the treatment of the edge phosphorus atoms.
\end{abstract}

Keywords: phosphorene, chemical scissors, phosphorus chains, phosphorene nanoribbons, Dirac material, Fermi velocity

\section{Introduction}

Two-dimensional (2D) few-layer black phosphorus and phosphorene structures [1-4] have attracted immediate research interest from the material science community [5-16]. The materials are chemically inert and have shown promising transport properties. They have carrier mobility up to $1000 \mathrm{~cm}^{2} \mathrm{~V}^{-1} \mathrm{~s}^{-1}$ and an on/off ratio up to $10^{4}$ was achieved for the phosphorene transistors at room temperature $[1,2]$. Moreover, these materials demonstrate a direct band gap at $\Gamma$ of the Brillouin zone $[1,2,5,7,17,18]$ (in contrast to the zero-gap in graphene), which creates potential for applications in optoelectronics. 
For practical applications, it is essential to enable lithography technique on 2D layered materials. For example, extensive research work has been carried out on graphene. Conventional approaches include e-beam lithography [19] by burning off material, plasma etching [20], lithography by atomic force microscopy and scanning tunneling microscopy $[21,22]$ etc. These methods involve aggressive physical/chemical forces, bringing the concern of introduction of undesired defects into the patterned structures.

Chemical functionalization of the surface has been widely explored on graphene for patterning purpose [23, 24]. One example is graphane-hydrogenated graphene [24-26]. In this case, the delocalized $\pi$ bond of carbon was broken with the introduction of a bond with hydrogen. This hydrogenated surface can be recovered by thermal heating, which makes a promising and controllable way for patterning and tuning the properties of graphene. Meanwhile, hydrogen surface passivation on graphene does not break the backbone of $\mathrm{C}-\mathrm{C}$ bonds and is not a direct method for cutting graphene nanostructures. However, hydrogen surface passivation on the $2 \mathrm{D}$ phosphorene demonstrates a distinct chemical scissors effect.

In this report, the effect of surface passivation on phosphorene was explored using firstprinciples density functional theory (DFT) calculations. It was found that some chemical species, for instance $\mathrm{H}, \mathrm{OH}, \mathrm{F}$ and $\mathrm{Cl}$, could dismantle the structure of phosphorene and fewlayer black phosphorus into nano-patterns, in addition to their traditional role of engineering electronic properties of the materials. With such surface passivation, some $\mathrm{P}-\mathrm{P}$ bonds in the phosphorene are literally broken in which those $\mathrm{P}-\mathrm{P}$ bond lengths are enlarged by at least $140 \%$ of their original value. The monolayer of black phosphorus has a rippled structure, which can be distinguished as upper and lower half-layer structures. With $\mathrm{H}$ (or $\mathrm{F}, \mathrm{Cl}, \mathrm{OH}$ radicals) passivation on the phosphorus atoms, $\mathrm{H}$ and $\mathrm{P}$ form a strong $\sigma$ bond and break the $\mathrm{P}-\mathrm{P}$ bonds between the upper and lower half-layers of phosphorene, which acts effectively as scissors to cut phosphorene. By selecting the location of surface passivation, different shape and size of phosphorene nanostructures, such as phosphorene nanoribbons (PNRs) or nanochains can be synthesized with a precise control.

Different experimental approaches may be employed to obtain the desired 'scissors' radicals from an appropriate chemical treatment. For relatively inert molecules such as $\mathrm{H}_{2} \mathrm{O}, \mathrm{H}$ and $\mathrm{OH}$ radicals might be produced by cracking thermally through heated filaments, or remote plasma, or plasma with very low energy ions. Fluorine and chlorine molecules would react directly with the phosphorene surface without particular physical assistance. The low dimensional nanostructures are expected to have wide potential applications [27-35], such as nanoscale transistors, sensors, optoelectronics and interconnect. Here it is demonstrated that the chemical scissor effects possess a possibility to obtain one-dimensional (1D) phosphorene nanostructures (nanoribbons and nanochains) for desired applications from the 2D planar structure.

It is also very interesting to find that the obtained pristine zigzag phosphorus-chain is a $1 \mathrm{D}$ Dirac material in which the charge carriers are massless fermions travelling at an effective speed of light $\sim 8 \times 10^{5} \mathrm{~m} \mathrm{~s}^{-1}$. This extraordinary electronic property of the pristine chain is essentially related to its topographic symmetry. In addition, the produced zigzag phosphorene nanoribbons are either metallic or semiconducting, depending on the treatment of the edge $\mathrm{P}$ atoms [36]. The ribbons are semiconductors if the dangling bonds of the edge $\mathrm{P}$ atoms are saturated and they show metallic behavior if the dangling bonds exist. 

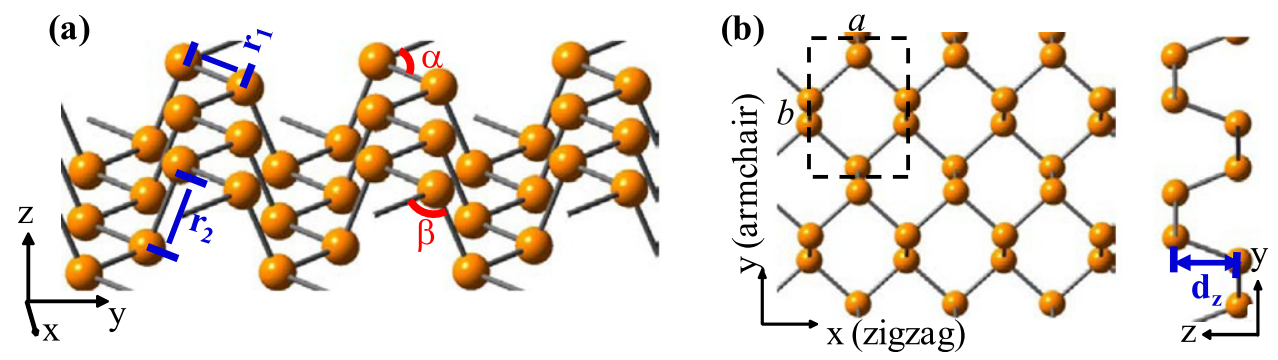

Figure 1. Snapshots of the 2D puckered phosphorene structure. The dashed rectangle in (b) indicates a unit cell.

\section{Methodology}

The ab initio calculations were carried out using first-principles density functional theory (DFT) [37]. The Perdew-Burke-Ernzerhof (PBE) exchange-correlation functional [38] and the projector-augmented wave (PAW) potentials $[39,40]$ were employed. The calculations were performed using the Vienna Ab-initio Simulation Package (VASP) [41, 42]. The electronic property of the pristine P-chain was validated using advanced functional HSE method [43, 44] and GW calculations $[45,46]$. The kinetic energy cutoff for the plane wave basis set was chosen to be $500 \mathrm{eV}$. The energy convergence criteria for electronic and ionic iterations were set to be $10^{-5} \mathrm{eV}$ and $10^{-4} \mathrm{eV}$, respectively. The reciprocal space for the unit cell of monolayer phosphorene was meshed at $14 \times 10 \times 1$ using Monkhorst Pack meshes centered at $\Gamma$ point. The non-spin-polarized calculations were used to relax the geometries of the systems. For the phosphorene nanoribbons, spin-polarized calculations were performed to calculate their electronic band structures and density of states. $21 \mathrm{~K}$-points were included in band structure calculations along $\Gamma$ to $\mathrm{X}$ for the zigzag P-chains and ribbons. A unit cell with periodic boundary condition was used. A vacuum space of at least $20 \AA$ was included in the unit cell to minimize the interaction between the system and its replicas resulting from the periodic boundary condition.

The initial structures of monolayer phosphorene were obtained from bulk black phosphorus [47]. Monolayer black phosphorus has a puckered honeycomb structure with each phosphorus atom covalently bonded with three adjacent atoms. Our calculated lattice constants for bulk black phosphorus are $a=3.307 \AA, b=4.547 \AA$, and $c=11.210 \AA$, in good agreement with experimental values [47] and other theoretical calculations [1, 48]. The relaxed lattice constants for a monolayer of phosphorene are $a=3.295 \AA$ and $b=4.618 \AA$.

\section{Results and discussion}

\subsection{Chemical scissors effect}

The 2D phosphorene structure is shown in figure 1. Phosphorene is puckered, unlike the flat graphene structure. The spatial distance $d_{\mathrm{z}}$ between the two half-layer $\mathrm{P}$ atoms is $2.10 \AA$. The bond length $r_{1}$ and bond angle $\alpha$ within a half-layer (in the xy-plane) are $2.22 \AA$ and $95.9^{\circ}$, respectively. The bond length $r_{2}$ and bond angle $\beta$ connecting $\mathrm{P}$ atoms on both half-layers are $2.26 \AA$ and $104.1^{\circ}$, respectively. 

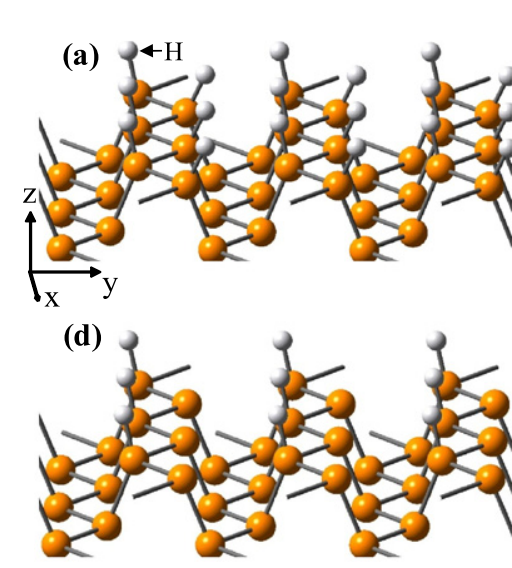

(g)

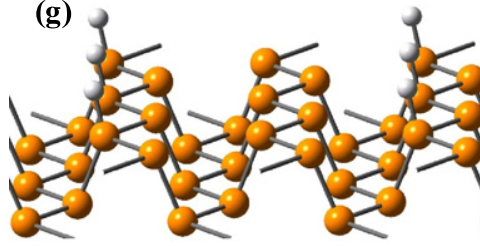

(b)

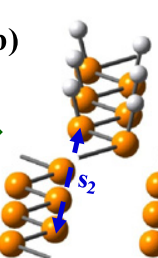

(e)

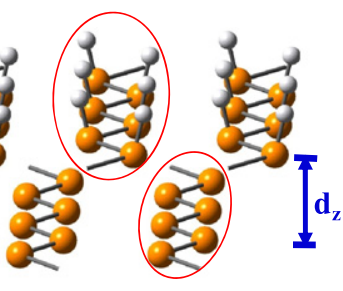

(c)

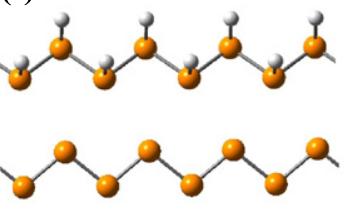

(f)

Figure 2. Chemical scissors effect of $H$ on phosphorene. (a), (d) and (g) are the initial structures with different $H$ surface coverage ratios of $H: P(1: 1,1: 2,1: 4$, respectively). (b), (e) and (h) are the DFT relaxed geometries. The products of the $\mathrm{H}$ scissors inside the ovals are displayed in (c) the hydrogenated and pristine zigzag P-chains, (f) the $4 \mathrm{~L}-\mathrm{ZPNR}$, and (i) the $8 \mathrm{~L}-\mathrm{ZPNR}$. The $\mathrm{H}$ scissors can be used to cut $n \mathrm{~L}-\mathrm{ZPNR}$ where $n=4 m$ with $m$ an integer. The orange and white dots represent $\mathrm{P}$ and $\mathrm{H}$ atoms, respectively.

$\mathrm{H}$ atoms were first added on the top half-layer with different surface coverage. For example, each $\mathrm{P}$ atom at the top half-layer was terminated with an $\mathrm{H}$ atom, which is denoted as surface coverage $\mathrm{H}: \mathrm{P}=1: 1$, as shown in figure 2(a). Note that the $\mathrm{P}$ atoms at the bottom halflayer were not treated with $\mathrm{H}$, considering the situation that the materials were on a substrate. To simulate a surface coverage smaller than 1 , supercells were employed. For instance, a $1 \times 2$ supercell of monolayer phosphorene was used to consider the surface coverage $\mathrm{H}: \mathrm{P}=1: 2$, in which $\mathrm{H}$ atoms were added to $50 \%$ of $\mathrm{P}$ atoms at the top half-layer (see figure 2(d)). Similarly, a $1 \times 4$ supercell was used to simulate the surface coverage of $1: 4$, and so on.

Figures 2(a), (d), and (g) gives the snapshot of the initial structures with the surface coverage of $1: 1,1: 2$, and $1: 4$, respectively. Periodic boundary conditions in the $\mathrm{x}$ and $\mathrm{y}$ directions and a vacuum space of $20 \AA$ in the $\mathrm{z}$ direction were employed and the configuration of the whole system was relaxed within the frame of DFT. Figures 2(b), (e), and (h) display their relaxed geometries. It is clear that the $\mathrm{H}$ atoms form bonds with $\mathrm{P}$ atoms and effectively break the P-P bonds (i.e., $r_{2}=2.26 \AA$ ) between the top and bottom half-layer of phosphorene. The resulting P-P distances $s_{2}$ are $3.55 \AA$, $3.29 \AA$, and $3.82 \AA$, in figures $2(\mathrm{~b})$, (e), and (h), respectively, which correspond to $157 \%, 146 \%$, and $169 \%$ increase, respectively, compared to that original $r_{2}$ value. In the case of coverage ratio 1:1 in figure 2(b), the full coverage of $\mathrm{H}$ on each $\mathrm{P}$ atoms breaks all $\mathrm{P}-\mathrm{P}$ bonds connecting the top and bottom half layers. The products of the $\mathrm{H}$ scissors are two types of zigzag $\mathrm{P}$-chains. The top gives a hydrogenated $\mathrm{P}$-chain and the bottom is a pristine $\mathrm{P}$-chain, as shown in figure 2(c). 
Table 1. The relaxed bond lengths and angles for the studied chemical species on monolayer phosphorene. The bond lengths and angles are denoted in figures 1 and 2. As a reference, the bond lengths/angles in monolayer phosphorene were also listed. The percentage values in the parentheses are the ratios of the corresponding values in the passivated phosphorene to that in the pristine monolayer.

\begin{tabular}{llllllll}
\hline System & Coverage ratio & $r_{1}(\AA)$ & $r_{2}$ or $s_{2}(\AA)$ & $d_{z}(\AA)$ & $\alpha\left(^{\circ}\right)$ & $\beta\left(^{\circ}\right)$ & $\gamma\left(^{\circ}\right)$ \\
\hline Monolayer & 0 & 2.22 & 2.26 & 2.10 & 95.9 & 104.1 & n/a \\
& $1: 1$ & 2.23 & $3.55(157 \%)$ & $3.43(164 \%)$ & 95.5 & n/a & 98.6 \\
& $1: 2$ & 2.25 & $3.29(146 \%)$ & $2.87(137 \%)$ & 94.4 & 96.5 & 93.2 \\
H:P & $1: 4$ & 2.26 & $3.82(169 \%)$ & $3.35(160 \%)$ & 93.9 & 93.9 & 92.0 \\
& $1: 6$ & 2.26 & $3.83(170 \%)$ & $3.36(160 \%)$ & 93.9 & 93.0 & 92.2 \\
& $1: 8$ & 2.26 & $3.86(171 \%)$ & $3.38(161 \%)$ & 93.8 & 92.6 & 91.9 \\
& $1: 10$ & 2.25 & $3.61(160 \%)$ & $3.19(152 \%)$ & 94.0 & 93.3 & 91.7 \\
F:P & $1: 6$ & 2.27 & $3.38(150 \%)$ & $3.15(150 \%)$ & 93.2 & 96.0 & 96.0 \\
Cl:P & $1: 6$ & 2.27 & $3.47(154 \%)$ & $3.19(152 \%)$ & 93.3 & 95.5 & 96.1 \\
OH:P & $1: 6$ & 2.26 & $3.43(152 \%)$ & $3.05(145 \%)$ & 93.1 & 92.8 & 94.7 \\
O:P & $1: 6$ & 2.24 & $2.24(99 \%)$ & $2.03(97 \%)$ & 95.0 & 98.9 & 112.6 \\
S:P & $1: 6$ & 2.24 & $2.25(100 \%)$ & $2.03(97 \%)$ & 94.7 & 100.1 & 112.7 \\
Se:P & $1: 6$ & 2.25 & $2.27(100 \%)$ & $1.94(92 \%)$ & 94.4 & 98.6 & 109.9 \\
\hline
\end{tabular}

However, for the coverage ratio 1:2 in figure 2(e), only 50\% of P-P bonds between the top and bottom half-layers were broken. This produces a narrow zigzag-PNR (ZPNR) with a width of $4.6 \AA$, in which one edge of the ribbon is saturated with $H$ and the other edge is pristine $P$ atoms. Similarly, for the coverage ratio 1:4 in figure 2(h), a quarter of $\mathrm{P}-\mathrm{P}$ bonds were broken by $\mathrm{H}$ and result in a relatively wider ZPNR with a width of $9.2 \AA$. The width of a ribbon can also be referred to as $n \mathrm{~L}$, according to the number $n$ of $\mathrm{P}$ atoms in the armchair direction (see figure 2(i)). As an example, figures 2(f) and (i) demonstrate the snapshots of 4L-ZPNR and 8LZPNR. Furthermore, a coverage ratio of 1:8 will generate a 16L-ZPNR. These $\mathrm{H}$ scissors can be used to cut $n \mathrm{~L}-\mathrm{ZPNR}$ (where $n=4 m$ with $m$ an integer) with a width $w=m^{*} 4.6 \AA$.

The bond lengths and angles for the geometrically relaxed structures with different $\mathrm{H}$ surface coverage ratios are listed in table 1. Compared to that of monolayer phosphorene, the bond length $r_{1}$ and bond angle $\alpha$ within a half-layer (in the xy-plane) have negligible changes. However, the bond length $r_{2}$ (or P-P distance $s_{2}$ ) and the spatial distance $d_{\mathrm{z}}$ between two halflayer $\mathrm{P}$ atoms show significant increases due to the scissors effect. For the varying surface coverage of $\mathrm{H}$ on the phosphorene, the $\mathrm{P}-\mathrm{P}$ bond distance $s_{2}$ was increased at least $146 \%$ and the spatial distance $d_{\mathrm{z}}$ was enlarged more than $137 \%$.

In addition to $\mathrm{H}$, we investigated other chemical species, such as $\mathrm{F}, \mathrm{Cl}, \mathrm{O}, \mathrm{S}, \mathrm{Se}$ and the $\mathrm{OH}$ group. It was found that $\mathrm{F}, \mathrm{Cl}$ and $\mathrm{OH}$ demonstrate a scissors effect similar to $\mathrm{H}$. However, adding $\mathrm{O}, \mathrm{S}$, and Se on the surface of phosphorene does not break the P-P bonds connecting the top and bottom half-layers. As an example, figure 3 displays their relaxed geometries with the surface coverage of 1:2. For the $\mathrm{O}(\mathrm{S}$ or $\mathrm{Se}$ ) case, we checked different initial configurations. For instance, one initial setup places $\mathrm{O}$ ( $\mathrm{S}$ or $\mathrm{Se}$ ) near the top of one $\mathrm{P}$ atom. Another one places $\mathrm{O}$ ( $\mathrm{S}$ or $\mathrm{Se}$ ) on the bridge of two $\mathrm{P}$ atoms. The former is energetically more favorable compared to the latter. The relaxed geometries of all configurations show that $\mathrm{O}(\mathrm{S}$ or $\mathrm{Se})$ does not break the P-P bonds where the variation of the P-P bond length is within $\pm 2 \%$ compared to its original value. 

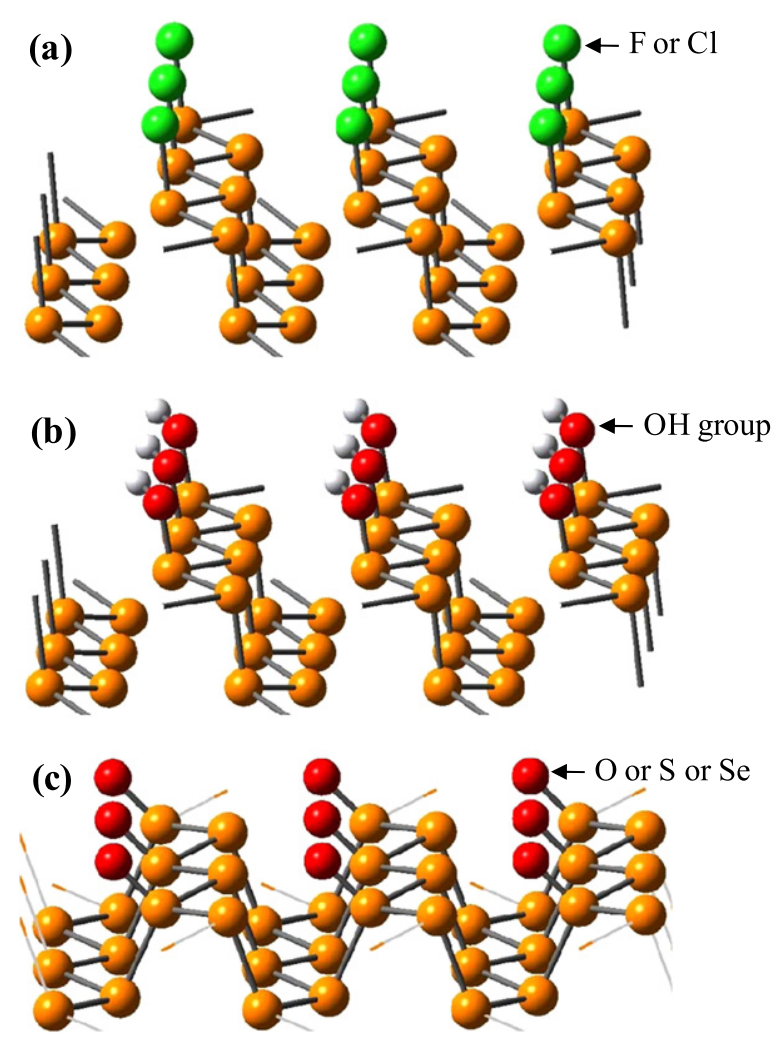

Figure 3. The snapshots of the DFT relaxed geometries of additional (a), (b) successful and (c) failed chemical scissors for phosphorene.

Table 1 also lists the geometry parameters such as bond lengths and angles for these additional species. For each chemical species, varying coverage ratios were examined similar to the $\mathrm{H}$ case. As an example, only 1:6 coverage ratio is listed for the species of $\mathrm{F}, \mathrm{Cl}, \mathrm{OH}, \mathrm{O}, \mathrm{S}$, and $\mathrm{Se}$ in table 1. Note that other coverage ratios have similar behaviors compared to this 1:6 ratio for each chemical species. Similar to $\mathrm{H}$, the bond distance $r_{2}\left(\right.$ or $\left.s_{2}\right)$ and the spatial distance $d_{\mathrm{z}}$ largely increase $(\sim 150 \%)$ for $\mathrm{F}, \mathrm{Cl}$ and $\mathrm{OH}$ surface passivation, when compared to that of monolayer phosphorene. However, in the cases of $\mathrm{O}, \mathrm{S}$ and $\mathrm{Se}, r_{2}$ and $d_{\mathrm{z}}$ are very close to the monolayer structure. It is meaningful to mention that the bond angle $\gamma$ (denoted in figure 2) has similar value $\left(92^{\circ} \sim 99^{\circ}\right)$ for the chemical species which show the scissors effect. However, for $\mathrm{O}, \mathrm{S}$ and Se, the bond angle $\gamma$ increases by about $20^{\circ}$. This results from their different structural geometries and electronic orbital orientations. As shown in figure 3(a), the P atoms form bonds with $\mathrm{F}(\mathrm{Cl})$ and their bonds with $\mathrm{P}$ at the bottom half-layer were broken. The $\mathrm{P}$ atoms still had three covalent bonds, among which the bond angles $\alpha, \beta$, and $\gamma$ were nearly $90^{\circ}$ due to their particular p-orbital orientations (i.e. $\mathrm{p}_{\mathrm{x}}, \mathrm{p}_{\mathrm{y}}$, and $\mathrm{p}_{\mathrm{z}}$ ). On the other hand, in figure 3(c), those $\mathrm{P}$ atoms formed four bonds; three were the original bonds with $\mathrm{P}$ and one additional with $\mathrm{O}$ ( $\mathrm{S}$ or $\mathrm{Se})$. This placed $\mathrm{P}$ in a nearly tetrahedral configuration and increased the bond angle $\gamma$ to $112^{\circ}$.

To further understand the chemical scissors effect, specifically why some chemical species work while others fail, we examined in detail the wavefunction and charge density of the electronic orbitals of the systems. It was found that whether a chemical species can effectively cut phosphorene depends on if it is strongly bonded to $\mathrm{P}$ atoms. For the cases of $\mathrm{H}, \mathrm{OH}, \mathrm{F}$, and $\mathrm{Cl}$, these species form a strong bond with $\mathrm{P}$; for example, the s-orbital of $\mathrm{H}$ atom bonds with the 
(a)

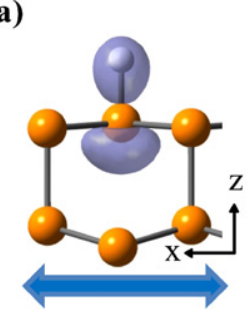

(d)

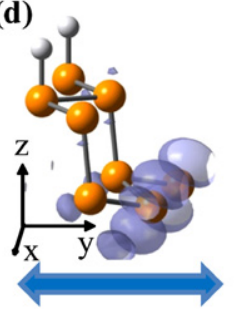

(b)

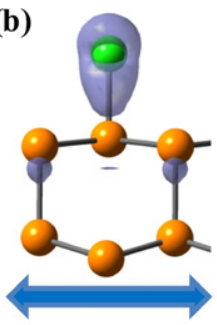

(e)

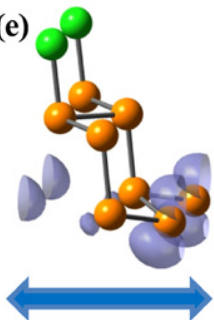

(c)

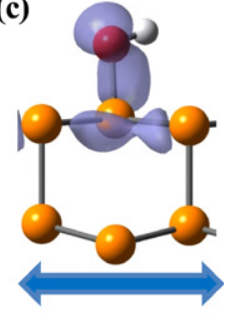

(f)

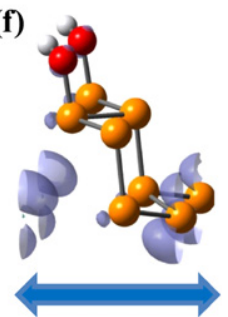

Figure 4. The electron density contour plots of the (a) $\mathrm{P}-\mathrm{H}$ (b) $\mathrm{P}-\mathrm{Cl}$ (c) $\mathrm{P}-\mathrm{O}$ bond for the chemical species of $\mathrm{H}, \mathrm{Cl}$, and $\mathrm{OH}$ group, respectively. The scissor effects result from their strong bonding with the $\mathrm{P}$ atoms. (d)-(f) The electron density of the nearFermi-level states contributed by the edge $\mathrm{P}$ atoms at the pristine side in each case. The horizontal blue arrows indicate the periodic boundary directions of the simulation cells. The orange, white, blue and red dots represent $\mathrm{P}, \mathrm{H}, \mathrm{Cl}$, and $\mathrm{O}$ atoms, respectively.

$\mathrm{p}_{\mathrm{z}}$-orbital of P. Figure 4(a) plots the electron density contour plot of the structure shown in figure 2(e) with both periodic boundaries in the $\mathrm{x}$ and $\mathrm{y}$ directions. The orbital energy of this $\mathrm{H}-$ $\mathrm{P}$ bond is $5.33 \mathrm{eV}$ below the Fermi level. Figure 4(b) shows a strong $\mathrm{p}_{\mathrm{z}}$-orbital bonding between $\mathrm{Cl}$ and $\mathrm{P}$ from the structure shown in figure 3(a) and its orbital energy is $5.23 \mathrm{eV}$ below the Fermi energy. Figure 4(c) displays the $\mathrm{p}_{\mathrm{z}}$-bond between $\mathrm{P}$ and $\mathrm{O}$ in the $\mathrm{OH}$ group from the structure displayed in figure 3(b), and the orbital energy is $6.08 \mathrm{eV}$ under the Fermi level. For the cases of $\mathrm{O}, \mathrm{S}$, and Se shown in figure 3(c), weaker bonding between $\mathrm{P}$ and these species was found.

Detailed analysis of the electronic structures (i.e. band structure and density of states) of the phosphorene nanoribbons resulting from the chemical scissor effects reveals that the ribbons show metallic behavior, in which the bands near the Fermi level are contributed by $\mathrm{P}$ atoms on the pristine edge [36]. To demonstrate this, we present the electron density of the near-Fermilevel states in figures 4(d)-(f) for the passivation species of $\mathrm{H}, \mathrm{Cl}$, and $\mathrm{OH}$, respectively. It is clear that the charge density is contributed by $\mathrm{P}$ atoms at the pristine side of the phosphorene ribbons. Note that the nanoribbons can be tuned to be semiconductors if the $\mathrm{P}$ atoms on the pristine edge were passivated using $\mathrm{H}, \mathrm{F}, \mathrm{Cl}$ or $\mathrm{OH}$ radicals [36] as demonstrated below in the study of density of states (DOS).

\subsection{Electronic properties of P-chains and ribbons}

We further calculated the electronic properties of the produced P-chains and nanoribbons from the chemical scissors. Figure 5 presents the band structure and DOS of the two P-chains. Left is the hydrogenated and right is the pristine zigzag P-chains. The hydrogenated chain is an insulator and the DFT predicted band gap is $3.82 \mathrm{eV}$. For other chemical species such as $\mathrm{Cl}$, and $\mathrm{OH}$, the DFT gaps are $2.78 \mathrm{eV}$ and $2.28 \mathrm{eV}$, respectively. 
(a)

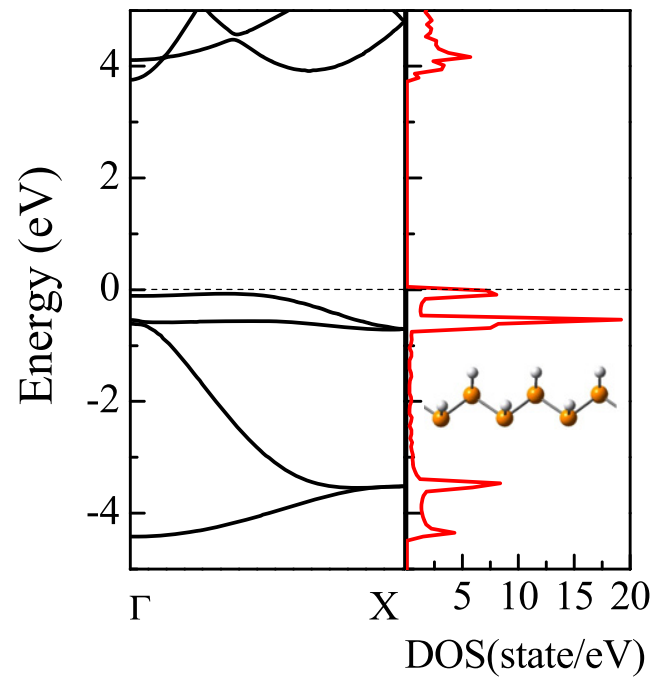

(b)

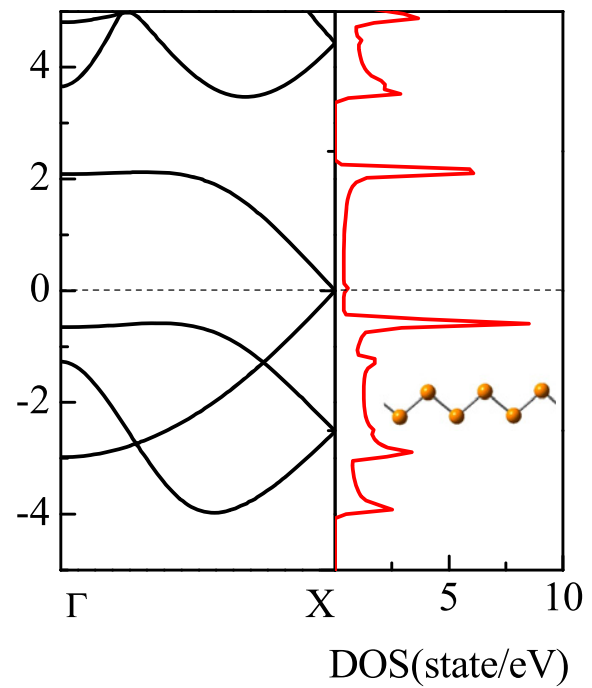

Figure 5. Band structure and density of states of the (a) hydrogenated and (b) pristine zigzag P-chains. The hydrogenated P-chain is an insulator with the DFT predicted gap of $3.82 \mathrm{eV}$. The pristine P-chain has zero band gap and demonstrates a Dirac point at X. The Fermi level is set at the valence band maximum which is aligned at zero. The insets are the snapshots of the corresponding P-chains.

However, the pristine P-chain in figure 5(b) shows zero gap at the $\mathrm{X}$ point. More interestingly, the dispersion curve of the conduction and valence bands near the $\mathrm{X}$ point demonstrate a linear relation, which makes $X$ a Dirac point for this structure. We used the advanced hybrid functional HSE method $[43,44]$ and GW calculations $[45,46]$ to check the band structure of the pristine P-chain and found that the Dirac point remains at X.

For the 1D Dirac material of the pristine zigzag P-chain, its Fermi velocity can be calculated as follows. The linear dispersion relation at X can be expressed as $E= \pm \beta k$, where $k$ is the distance of wave vector away from the point $\mathrm{X}$ and the slope $\beta$ can be fitted from the first principles calculations. According to the relativistic energy-momentum relationship $E=\sqrt{m_{0}^{2} c^{4}+p^{2} c^{2}}$, the linear dispersion relation at $\mathrm{X}$ implies that the charge carriers are massless fermions traveling at an effective speed of light $v$ (Fermi velocity): $E=v p=v \hbar K=v \hbar k 2 \pi / a=v h k / a$, where $a$ is the lattice constant of the pristine zigzag Pchain. Therefore, the Fermi velocity $v$ can be calculated from the expression $v=\beta a / h$. Our calculated Fermi velocity for the pristine P-chain is $\sim 8 \times 10^{5} \mathrm{~m} \mathrm{~s}^{-1}$.

Figure 6 displays the DOS for the ZPNRs with different widths, namely 4L-ZPNR $(w=4.6 \AA)$ and 12L-ZPNR ( $w=13.8 \AA)$. Figures $6(\mathrm{a})$ and $(\mathrm{b})$ are their corresponding plots of the DOS. They show either half-metallic or metallic behaviors. The bands that cross the Fermi level are contributed by the edge $\mathrm{P}$ atoms on the pristine side which is clearly demonstrated by the electron density plot in figure 4(d). Adding $\mathrm{H}$ to those $\mathrm{P}$ atoms on the pristine side will open the band gap [36]. Figures 6(c) and (d) are the DOS for the 4L-PNR and 12L-ZPNR with both edges saturated using $\mathrm{H}$. The ribbons are semiconductors and the band gap reduces with the increase of the ribbon width due to quantum confinement. 
(a)

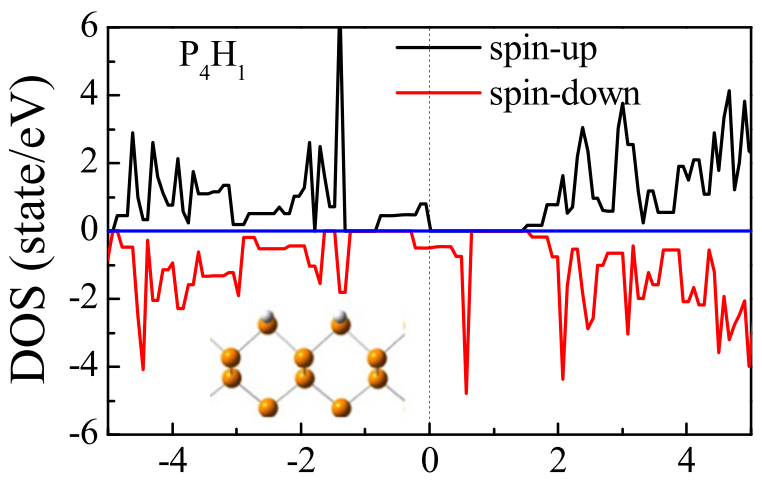

(c)

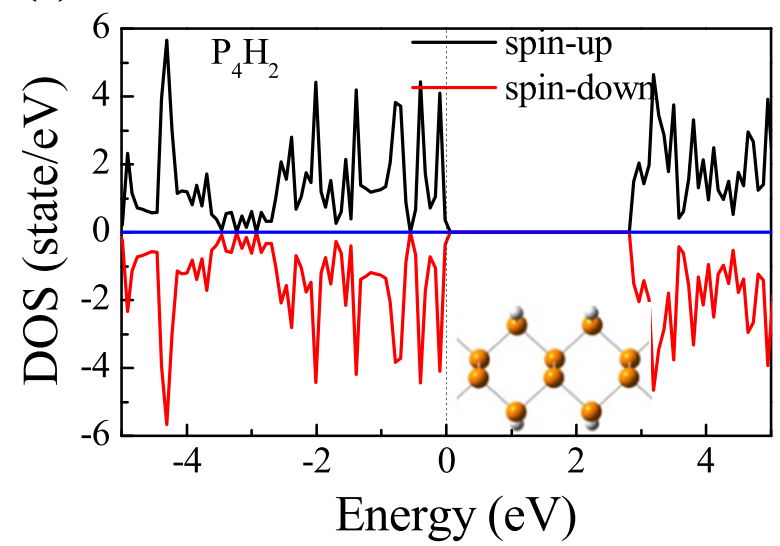

(b)

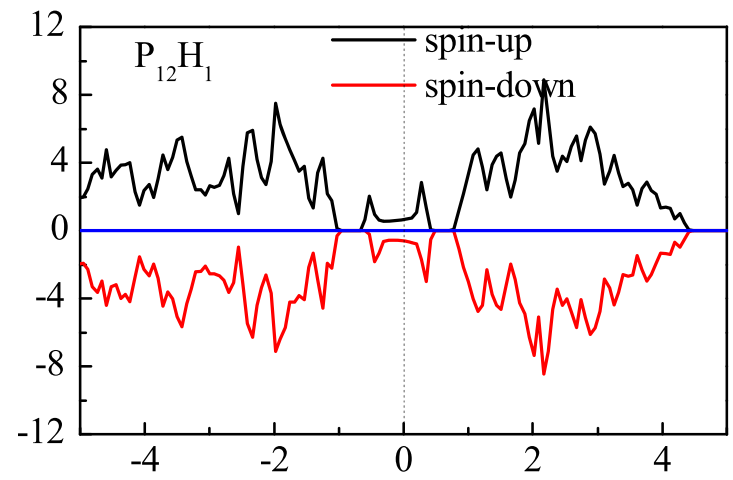

(d)

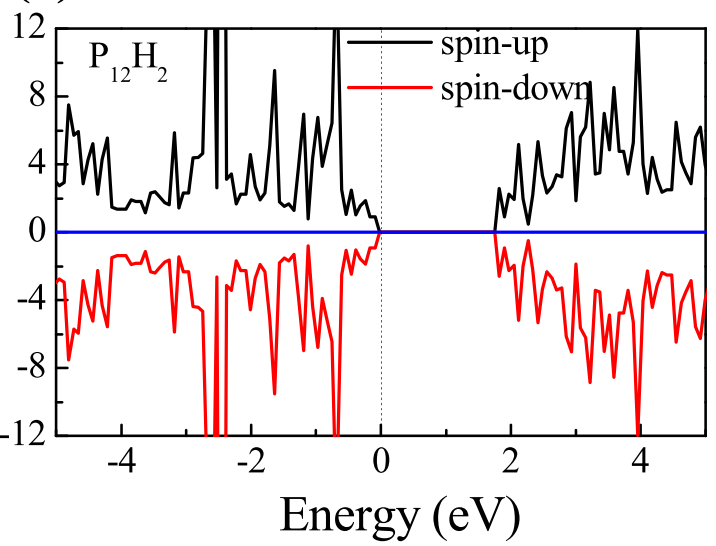

Figure 6. DOS of the (a) 4L-ZPNR and (b) 12L-ZPNR with one edge passivated with $\mathrm{H}$ and the other edge pristine. DOS of the (c) 4L-ZPNR and (d) 12L-ZPNR with both edges saturated with H. The Fermi level is set at zero. The insets in (a) and (c) are the snapshots of the corresponding 4L-ZPNRs.

\section{Summary}

We employed first-principles DFT calculations to study the chemical scissors effect on phosphorene. We found that several chemical species such as $\mathrm{H}, \mathrm{OH}, \mathrm{F}$, and $\mathrm{Cl}$ can be used as chemical scissors to cut phosphorene, while $\mathrm{O}, \mathrm{S}$ and Se cannot, depending on if the species forms a strong chemical bond with P. Phosphorus chains and nanoribbons can be produced using these chemical scissors. The saturated P-chain is insulating, while the pristine P-chain is a 1D Dirac material with the Fermi velocity about $8 \times 10^{5} \mathrm{~m} \mathrm{~s}^{-1}$. The zigzag nanoribbons are either metallic or semiconducting, depending on the treatment of the edge $\mathrm{P}$ atoms. The ribbons are semiconductors if the dangling bonds of the edge $\mathrm{P}$ atoms are saturated. They show metallic behavior if dangling bonds exist.

\section{Acknowledgements}

This work is supported by the Faculty Research Fund from School of Letters and Sciences at Arizona State University (ASU) to Peng. The authors thank ASU Advanced Computing Center 
and XSEDE for providing computing resources. Dr F Tang is acknowledged for the helpful discussions and we thank A Copple for his critical review of the manuscript.

\section{References}

[1] Liu H, Neal A T, Zhu Z, Luo Z, Xu X, Tománek D and Ye P D 2014 ACS Nano 84033

[2] Li L, Yu Y, Ye G J, Ge Q, Ou X, Wu H, Feng D, Chen X H and Zhang Y 2014 Nat. Nanotechnol. 9372

[3] Xia F, Wang H and Jia Y 2014 Nat. Commun. 54458

[4] Reich E S 2014 Nature 50619

[5] Tran V, Soklaski R, Liang Y and Yang L 2014 Phys. Rev. B 89235319

[6] Fei R and Yang L 2014 Nano Lett. 142884

[7] Peng X, Copple A and Wei Q 2014 Phys. Rev. B 90085402

[8] Dai J and Zeng X C 2014 J. Phys. Chem. Lett. 51289

[9] Guo H, Lu N, Dai J, Wu X and Zeng X C 2014 J. Phys. Chem. C 11814051

[10] Wei Q and Peng X 2014 Appl. Phys. Lett. 104251915

[11] Lu W, Nan H, Hong J, Chen Y, Zhu C, Liang Z, Ma X, Ni Z, Jin C and Zhang Z 2014 Nano Res. 7853

[12] Tran V and Yang L 2014 Phys. Rev. B 89245407

[13] Maity A, Singh A and Sen P 2014 (arXiv:1404.2469)

[14] Carvalho A, Rodin A S and Neto A H C 2014 (arXiv:1404.5115)

[15] Lv H Y, Lu W J, Shao D F and Sun Y P 2014 (arXiv:1404.5171)

[16] Buscema M, Groenendijk D J, Blanter S I, Steele G A, van der Zant H S J and Castellanos-Gomez A 2014 Nano Lett. 143347

[17] Takao Y and Morita A 1981 Physica B \& C 10593

[18] Rodin A S, Carvalho A and Neto A H C 2014 Phys. Rev. Lett. 112176801

[19] Fischbein M D and Drndić M 2008 Appl. Phys. Lett. 93113107

[20] Bai J, Duan X and Huang Y 2009 Nano Lett. 92083

[21] Tapaszto L, Dobrik G, Lambin P and Biro L P 2008 Nat. Nanotechnol. 3397

[22] Weng L, Zhang L, Chen Y P and Rokhinson L P 2008 Appl. Phys. Lett. 93093107

[23] Zhang L, Diao S, Nie Y, Yan K, Liu N, Dai B, Xie Q, Reina A, Kong J and Liu Z 2011 J. Am. Chem. Soc. 1332706

[24] Feng J, Li W, Qian X, Qi J, Qi L and Li J 2012 Nanoscale 44883

[25] Elias D C et al 2009 Science 323610

[26] Sessi P, Guest J R, Bode M and Guisinger N P 2009 Nano Lett. 94343

[27] Novoselov K S, Geim A K, Morozov S V, Jiang D, Zhang Y, Dubonos S V, Grigorieva I V and Firsov A A 2004 Science 306666

[28] Novoselov K S, Geim A K, Morozov S V, Jiang D, Katsnelson M I, Grigorieva I V, Dubonos S V and Firsov A A 2005 Nature 438197

[29] Geim A K and Novoselov K S 2007 Nat. Mater. 6183

[30] Hirschman K D, Tsybeskov L, Duttagupta S P and Fauchet P M 1996 Nature 384338

[31] Cui Y and Lieber C M 2001 Science 291851

[32] Cui Y, Zhong Z H, Wang D L, Wang W U and Lieber C M 2003 Nano Lett. 3149

[33] Koo S M, Fujiwara A, Han J P, Vogel E M, Richter C A and Bonevich J E 2004 Nano Lett. 42197

[34] Cui Y, Wei Q Q, Park H K and Lieber C M 2001 Science 2931289

[35] Hahm J and Lieber C M 2004 Nano Lett. 451

[36] Peng X, Wei Q and Copple A 2014 J. Appl. Phys. 116144301

[37] Kohn W and Sham L J 1965 Phys. Rev. 140 A1133

[38] Perdew J P, Burke K and Ernzerhof M 1996 Phys. Rev. Lett. 773865

[39] Blochl P E 1994 Phys. Rev. B 5017953 
[40] Kresse G and Joubert D 1999 Phys. Rev. B 591758

[41] Kresse G and Furthmuller J 1996 Phys. Rev. B 5411169

[42] Kresse G and Furthmuller J 1996 Comput. Mater. Sci. 615

[43] Heyd J, Scuseria G E and Ernzerhof M 2003 J. Chem. Phys. 1188207

[44] Heyd J, Scuseria G E and Ernzerhof M 2006 J. Chem. Phys. 124219906

[45] Hedin L 1965 Phys. Rev. 139 A796

[46] Shishkin M and Kresse G 2006 Phys. Rev. B 74035101

[47] Brown A and Rundqvist S 1965 Acta Cryst. 19684

[48] Qiao J, Kong X, Hu Z X, Yang F and Ji W 2014 Nat. Commun. 54475 